ELK ASIA PACIFIC JOURNAL OF COMPUTER SCIENCE AND INFORMATION SYSTEMS

ISSN: 2454-3047; ISSN: 2394-0441 (Online) Volume 2 Issue 1 (2016)

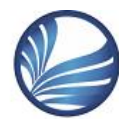

ELK

Asia Pacific Journals

www.elkjournals.com

\title{
SURVEY ON BRAIN TUMOUR DETECTION AND CLASSIFICATION USING IMAGE PROCESSING
}

\begin{tabular}{|c|c|c|}
\hline G.Santhosh Krishnan & K.Sivanarulselvan & P.Betty \\
P.G student & Associate Professor & Associate Professor \\
Department of computer science & Department of computer science & and engineering \\
and engineering & Department of computer science \\
and engineering \\
Kumaraguru college of technology \\
Coimbatore & Kumaraguru college of technology & Kumaraguru college of technology \\
India & Coimbatore & Coimbatore \\
India & India \\
santhoshkrish123@gmail.com & sivanarulsevan.k.cse@kct.ac.in & betty.p.cse@kct.ac.in \\
\hline
\end{tabular}

Radiology is a area in medical field where images are used to diagnose and treat various diseases seen within the body. Medical image processing is used to assist the physicians in diagnosing the disease. The brain tumour detection Brain tumor detection has gained importance in medical field because it provides anatomical information of abnormal tissues in brain which helps the doctors in planning the treatment. For brain tumour detection MRI and CT scans are used. Here in this paper median filter has been used to remove noise from the image, GLCM is used to extract the needed features from the image so that we can find whether the tumour is present or not, fuzzy c-means is used for image segmentation and artificial neuro fuzzy technology ANFIS is used for classification of type of brain tumour.

Keywords: Feature Extraction, Image Preprocessing, Image Segmentation, Image Classification, Neuro Fuzzy.

\section{Introduction}

The brain tumour is nothing but the growth of cells in brain that multiplies in an uncontrollable, abnormal way [11]. It can either be malignant or benign. Brain tumours are graded from 1 to 4 according to their behaviour, such as how quickly they grow and how likely they grow back after treatment. Radiology is a medical practice that makes use of imaging to diagnose and treat diseases [12]. Radiologists make use of techniques such as X-ray radiography, 


\section{ELK ASIA PACIFIC JOURNAL OF COMPUTER SCIENCE AND INFORMATION SYSTEMS}

ISSN: 2454-3047; ISSN: 2394-0441 (Online) Volume 2 Issue 1 (2016)

ultrasound, tomography, nuclear medicine along with positron emission tomography, and magnetic resonance imaging to diagnose diseases. Interventional radiology is performance of the medical procedures with guidance of imaging technologies.

The acquiring of medical imaging is carried out with the help of radiographer, often known as a radiologic technologist. Depending on position, the diagnostic radiologist interprets the images and produces a report of their diagnosis. The report is then transmitted to the doctor who ordered the imaging emergently. The Imaging exams are stored digitally in the picture archiving where they can be viewed by the members of the healthcare team who are in the same health system and related later on with future imaging exams.

\subsection{Magnetic Resonance Imaging (MRI)}

A strong magnetic field is first applied and then radio waves are applied for a short time in a different direction in magnetic resonance imaging. This sudden shift causes the certain atoms in patient's body to make special signals. The MRI scanner then detects those special signals caused by the radiation. The MRI scanner then sends the signal information to the computer, and then the computer creates an image of the inner body with the help of signal information.

\subsection{Types Of Neuro-Fuzzy Systems}

The Types of Neuro-Fuzzy Systems In general, all the combinations of the technique based on the neural networks and fuzzy logic can be called neuro-fuzzy systems. The different combinations of these techniques can be divided, in accordance with the following classes: Cooperative Neuro-Fuzzy System: In cooperative systems there is a phase called pre-processing phase where the neural network mechanisms of learning determine some sub-blocks of the fuzzy system. Fuzzy sub-blocks are calculated and the neural network learning methods are taken away, executing only fuzzy system. Concurrent Neuro-Fuzzy System: In the concurrent systems the neural network and the fuzzy system work continuously together. In general, neural networks pre-processes the inputs of the fuzzy system. Hybrid NeuroFuzzy System: In Hybrid Neuro-Fuzzy System, a neural network is used to learn some parameters of the fuzzy system of the fuzzy system in an iterative way.

\section{Related Work}




\section{ELK ASIA PACIFIC JOURNAL OF COMPUTER SCIENCE AND INFORMATION SYSTEMS}

ISSN: 2454-3047; ISSN: 2394-0441 (Online) Volume 2 Issue 1 (2016)

Ketan Machhale et al. (2015) [7] proposed an intellectual classification system to recognize the normal and abnormal MRI brain images. At present, decision and the treatment of brain tumors is based on the symptoms and radiological appearance. Magnetic resonance imaging (MRI) is the very important controlled tool for anatomical judgment of the tumors in brain. Nowadays, various techniques were used for the classification of the brain cancer. Under these techniques used the modules like image preprocessing, image segmentation, image feature extraction and subsequent classification of brain cancer are performed. Support Vector Machine (SVM), K- Nearest Neighbor (KNN) and Hybrid Classifier (SVM-KNN) are the various machine learning techniques are used.

Trung Le et al. (2010) [10] proposed the new support vector machine technique for the two-class medical image classification. The main idea of the method is to construct an optimal hypersphere such that both the interior margin between the surface of this sphere, the normal data, and the exterior margin between this surface and the abnormal data are as large as possible. The proposed method is implemented easily and can reduce both the false positive and also false negative error rates to obtain very good classification results. The Support Vector Machine (SVM) classifier is a good classifier that works well on the wide range of classification problems, even problems in the high dimensions and the cases that are not linearly separable. Perhaps the biggest problem with the support vector approach is in choice of the kernel.

Swarnalatha et al. (2013) [9] proposed a concept based on the novel fuzzy approach with bit plane FCMBP approach. The bit plane filtering method is used to slice the presented image for classification to find out destroyed region of the presented image. The sliced image must be normalized with old techniques and then compared with fuzzy technique for the better classification and the cluster of the spoiled portion. Thereby control points are extracted that are further needed for reconstruction of the images. The performance of the fuzzy approach with bit plane technique is evaluated with the help of simulation and it is found that our approach yields better results when compared to other accessible methods. Its disadvantage is Only efficient for most significant bit-planes (MSB).

Zehra Karhan et al. (2015) [3] proposed a method that is used for determining whether 


\section{ELK ASIA PACIFIC JOURNAL OF COMPUTER SCIENCE AND INFORMATION SYSTEMS}

ISSN: 2454-3047; ISSN: 2394-0441 (Online) Volume 2 Issue 1 (2016)

the medical image belongs to that class or not, using textural features of the medical images. The study was conducted on the images in the IRMA (Image Retrieval in Medical Applications), in the international database. After performing the preprocess on medical images, discrete wavelet transform (DWT) was applied and then the discrete cosine transform (DCT) was applied to the each band components. After extrating the features, using of $1 \%, 3 \%, 5 \%$ and $7 \%$ of the obtained data were classified. K-Nearest neighbor algorithm was used in classification phase. The classificaiton performance was around the 87 persentage. One of the main highlight of the wavelets is that they offer a simultaneous localization in time and the frequency domain.

Parveen et al. (2015) [4] proposed a new hybrid technique based on support vector machine (SVM) and the fuzzy c-means for brain tumor classification. This algorithm is an combination of support vector machine (SVM) and fuzzy c-means, a hybrid technique for prediction of the brain tumor. In this algorithm image is enhanced with the help of techniques such as contrast improvement, and mid-range stretch. Double thresholding and the morphological operations are used for the skull striping. The
Fuzzy c-means (FCM) clustering is used for the segmentation of image to detect suspicious region in the brain MRI image. Grey level run length matrix (GLRLM) is used for the extraction of the feature from the brain MRI image, after which the SVM method is used to classify brain MRI images, which provides accurate and more effective results for the classification of brain MRI images.

Walaa Hussein Ibrahim et al. (2013) [5] proposed the Neural Network techniques for the classification of magnetic resonance brain images. The proposed technique which uses neural network consists of three stages, preprocessing, dimensionality reduction, and the classification. In first stage, MR image will be obtained and then image is converted into data form. Then in the second stage the dimensionally reduction is obtained using principles component analysis (PCA), then In the classification stage Back-Propagation Neural Network has been used as a classifier to classify as normal or abnormal MRI brain images. The main disadvantage of this method is the training of the ANN is time taking.

\section{Conclusion}




\section{ELK ASIA PACIFIC JOURNAL OF COMPUTER SCIENCE AND INFORMATION SYSTEMS}

ISSN: 2454-3047; ISSN: 2394-0441 (Online) Volume 2 Issue 1 (2016)

The essential concepts of medical image processing are analyzed, specifically MRI brain images are considered. The study focus on the detection of the brain abnormalities and tumor from the MRI images. The related work First focuses on various image preprocessing methods for improvising the medical images, then segmentation types to effectively segment tumor parts from rest of the brain and then the feature extraction methods are studied. The final part is classification and this survey aims to analyze how the extracted features which can be used to classify whether the image comes under normal category or abnormal category. If it comes under the abnormal category, the brain tumour is present and if it comes under normal category, the brain tumour is not present.

\section{References}

[1] Hari Babu, Nandpuru, V. R.

Bora.(2014).MRI Brain Cancer

Classification Using Support Vector

Machine.IEEE Students Conference on

Electrical, Electronics and Computer

Science.

[2] Zuchita Goswami ,Latit Kumar P.Bhaiya.(2013). Brain tumour detection using unsupervised learning based neural network. international conference on communication systems and network technologies 2013.

[3] Zehra Karhan, Burhan Ergen.(2015).

Content Based Medical Image Classification Using Discrete Wavelet and Cosine Transforms. IEEE.

[4] Parveen, Amritpal Singh.(2015).

Detection of Brain Tumor in MRI Images, using Combination of Fuzzy C-Means and SVM. 2nd International Conference on Signal Processing and Integrated Networks (SPIN) .

[5] Walaa Hussein Ibrahim, Ahmed Abdel Rhman Ahmed Osman, Yusra Ibrahim Mohamed.(2013). MRI Brain Image Classification Using Neural Networks. international conference on computing, electrical and electronics engineering (icceee) 2013.

[6] Qing Li, Weidong Cai.(2014) Medical Image Classification with Convolutional Neural Network. 13th International Conference on Control, Automation, Robotics \& Vision Marina Bay Sands, Singapore (ICARCV). 


\section{ELK ASIA PACIFIC JOURNAL OF COMPUTER SCIENCE AND INFORMATION SYSTEMS}

ISSN: 2454-3047; ISSN: 2394-0441 (Online) Volume 2 Issue 1 (2016)

Computing, Communication and

[7] Ketan Machhale Hari Babu

Nanotechnology (ICECCN).

Nandpuru(2015) MRI Brain Cancer

Classification Using Hybrid Classifier

(SVM-KNN). International Conference on

[10] Trung Le, Dat Tran, Wanli Ma and

Dharmendra Sharma.(2010) a new support

Industrial Instrumentation and Control

(ICIC) .

vector machine method for medical image classification. ieee.

[8] Megha. P. Arakeri G. Ram Mohana

[11] What you need to know about tm brain

Reddy (2012)...Medical Image Retrieval tumors. u.s. department of health and human System for Diagnosis of the Brain Tumor services.

Based on Classification and Content

Similarity. IEEE .

[12] D.R. Dance S. Christofides . Diagnostic Radiology Physics A Handbook for

[9] Swarnalatha , B.K. Tripathy(2013). A Teachers and Students. international

Novel Fuzzy C-Means Approach with Bit automic energy agency.

Plane Algorithm for Classification of

Medical Images. IEEE International

[13] About brain tumours a primer for

Conference on Emerging Trends in patients and caregivers. American brain tumour association. 
ELK ASIA PACIFIC JOURNAL OF COMPUTER SCIENCE AND INFORMATION SYSTEMS

ISSN: 2454-3047; ISSN: 2394-0441 (Online) Volume 2 Issue 1 (2016)

\section{List of figures}

Figure 1: Hybrid Classifier

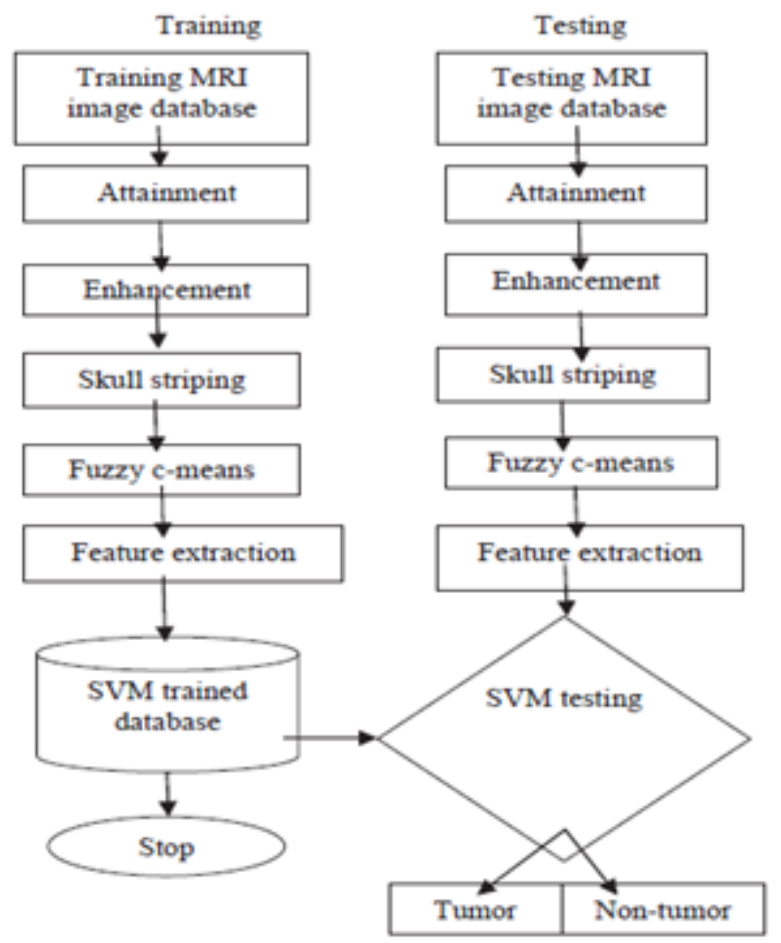

\section{List of Tables}


Table 1: Comparison

\begin{tabular}{|c|c|c|}
\hline $\begin{array}{l}\text { Classification } \\
\text { Method }\end{array}$ & Description & Characteristic Features \\
\hline $\begin{array}{l}\text { Artificial Neural } \\
\text { network }\end{array}$ & $\begin{array}{l}\text { ANN is a type of artificial intelligence that } \\
\text { imitates some functions of the person } \\
\text { mind. ANN has the normal tendency for } \\
\text { storing experiential knowledge. The ANN } \\
\text { consists of the sequence of layers, each } \\
\text { layer consists of a set of neurones. All the } \\
\text { neurones of each layer are linked by } \\
\text { weighted connections to all neurones on } \\
\text { the preceding and succeeding layers. }\end{array}$ & $\begin{array}{l}\text { It uses Nonparametric approach. } \\
\text { Performance and accuracy depends } \\
\text { upon the network structure and } \\
\text { number of inputs }\end{array}$ \\
\hline Fuzzy Measure & $\begin{array}{l}\text { In Fuzzy classification, various stochastic } \\
\text { associations are determined to describe } \\
\text { characteristics of image. The various types } \\
\text { of stochastic are combined in which } \\
\text { members of this set of properties have fuzzy } \\
\text { nature. It provides opportunity to describe } \\
\text { various categories of stochastic } \\
\text { characteristics in similar form }\end{array}$ & $\begin{array}{l}\text { It uses the Stochastic approach. } \\
\text { Performance and accuracy depends } \\
\text { upon the threshold selection and } \\
\text { fuzzy integral. }\end{array}$ \\
\hline Decision tree & $\begin{array}{l}\text { DT calculates class membership by } \\
\text { repeatedly partitioning a dataset into } \\
\text { uniform subsets Hierarchical classifier } \\
\text { permits acceptations and rejection of the } \\
\text { class labels at each intermediary stage. } \\
\text { This method consists of } 3 \text { parts: } \\
\text { Partitioning the nodes, find the terminal } \\
\text { nodes and allocation of class label to } \\
\text { terminal nodes }\end{array}$ & $\begin{array}{l}\text { DT are based on hierarchical rule } \\
\text { based method and use } \\
\text { Nonparametric approach. }\end{array}$ \\
\hline $\begin{array}{l}\text { Support Vector } \\
\text { Machine }\end{array}$ & $\begin{array}{l}\text { A support vector machine first builds a } \\
\text { hyper plane or set of hyper planes in an } \\
\text { high- or infinite dimensional space, used } \\
\text { for the classification. Better separation is } \\
\text { achieved by hyper plane that has largest } \\
\text { distance to nearest training data point of } \\
\text { any class. Generally larger the margin then } \\
\text { lower the generalization error of classifier. }\end{array}$ & $\begin{array}{l}\text { SVM makes use of Nonparametric } \\
\text { with binary classifier approach and } \\
\text { it can handle more input data very } \\
\text { efficiently. Performance and } \\
\text { accuracy depends upon the } \\
\text { hyperplane selection and kernel } \\
\text { parameter. Result transparency is } \\
\text { low. Training is time consuming }\end{array}$ \\
\hline
\end{tabular}

